\title{
Upregulation of Key Molecules for Targeted Imaging and Therapy
}

\author{
Vincent F. Taelman*1,2, Piotr Radojewski*1,2 ${ }^{*}$ Nicolas Marincek $^{1,2}$, Anat Ben-Shlomo ${ }^{3}$, Andrea Grotzky ${ }^{1,2}$, \\ Cristina I. Olariu ${ }^{1,2}$, Aurel Perren ${ }^{4}$, Christoph Stettler ${ }^{5}$, Thomas Krause ${ }^{1,2}$, Lorenz P. Meier ${ }^{1,2}$, Renzo Cescato ${ }^{1,2}$, \\ and Martin A. Walter ${ }^{1,2,6}$ \\ ${ }^{1}$ Department of Nuclear Medicine, Inselspital, University Hospital Bern, Bern, Switzerland; ${ }^{2}$ Clinical Radiopharmacy, Department of \\ Clinical Research, University Hospital Bern, Bern, Switzerland; ${ }^{3}$ Pituitary Center, Department of Medicine, Cedars Sinai Medical \\ Center, Los Angeles, California; ${ }^{4}$ Institute of Pathology, University Bern, Bern, Switzerland; ${ }^{5}$ Division of Diabetes, Endocrinology, \\ Nutritional Medicine \& Metabolism, University Hospital and Inselspital, Bern, Switzerland; and ${ }^{6}$ Molecular and Medical \\ Pharmacology, David Geffen School of Medicine, UCLA, Los Angeles, California
}

\begin{abstract}
Targeted diagnosis and therapy enable precise tumor detection and treatment. Successful examples for precise tumor targeting are diagnostic and therapeutic radioligands. However, patients with tumors expressing low levels of the relevant molecular targets are deemed ineligible for such targeted approaches. Methods: We performed a screen for drugs that upregulate the somatostatin receptor subtype 2 $\left(\mathrm{sstr}_{2}\right)$. Then, we characterized the effects of these drugs on transcriptional, translational, and functional levels in vitro and in vivo. Results: We identified 9 drugs that act as epigenetic modifiers, including the inhibitor of DNA methyltransferase decitabine as well as the inhibitors of histone deacetylase tacedinaline and romidepsin. In vitro, these drugs upregulated $\mathrm{sstr}_{2}$ on transcriptional, translational, and functional levels in a time- and dose-dependent manner. Thereby, their combinations revealed synergistic effects. In vivo, drug-based $\mathrm{sstr}_{2}$ upregulation improved the tumor-to-background and tumor-tokidney ratios, which are the key determinants of successful sstr ${ }_{2}-$ targeted imaging and radiopeptide therapy. Conclusion: We present an approach that uses epigenetic modifiers to improve sstr $_{2}$ targeting in vitro and in vivo. Translation of this method into the clinic may potentially convert patients ineligible for targeted imaging and therapy to eligible candidates.
\end{abstract}

Key Words: molecular imaging; PRRT; DOTATOC; DOTATATE; neuroendocrine tumors

J Nucl Med 2016; 57:1805-1810

DOI: $10.2967 /$ jnumed.115.165092

\section{$\mathbf{S}$} and therapy of somatostatin receptor subtype $2\left(\mathrm{sstr}_{2}\right)$-expressing malignancies such as neuroendocrine tumors and meningiomas (1-4). sstr $_{2}$-targeted imaging identifies tumors undetectable with conventional imaging modalities $(5,6)$, and $\operatorname{sstr}_{2}$-targeted therapy achieves responses in tumors that failed all previous treatments (7).

Received Aug. 4, 2015; revision accepted Apr. 22, 2016.

For correspondence or reprints contact: Martin A. Walter, University Hospital, $\mathrm{CH}-3010$ Bern, Switzerland.

E-mail: m.a.walter@gmx.net

${ }^{*}$ Contributed equally to this work.

Published online Jun. 30, 2016.

COPYRIGHT (C 2016 by the Society of Nuclear Medicine and Molecular Imaging, Inc.
However, several patients are not eligible for these targeted approaches. Patients with neuroendocrine tumors or meningiomas that express marginal $\operatorname{sstr}_{2}$ levels $(8)$ or that lost $\operatorname{sstr}_{2}$ expression during tumor progression (9), or patients with other tumor entities that normally express low $\operatorname{sstr}_{2}$ levels, such as prostate cancer (10), currently cannot benefit from $\mathrm{sstr}_{2}$-targeted imaging and therapy.

Herein, we describe a method to enable targeted therapies for these patients. We screened for drugs that upregulate $\operatorname{sstr}_{2}$ (Fig. 1), characterized their efficacy in vitro (Fig. 2), assessed synergistic effects (Fig. 3), and validated the upregulating effects in vivo (Fig. 4).

For these studies, we tracked and quantified the biodistribution and pharmacokinetics of radiolabeled somatostatin analogs and were thereby able to monitor changes in the expression of the relevant target, $\operatorname{sstr}_{2}$.

\section{MATERIALS AND METHODS}

All reagents and antibodies that we used are listed in the supplemental materials, together with a more detailed description of all experimental procedures (supplemental materials are available at http://jnm.snmjournals.org).

\section{Cell Models}

We used the human pancreatic neuroendocrine tumor cells BON-1, which express $\operatorname{sstr}_{2}$ at low to medium levels (11); the human prostate cancer cells PC3, which also express $\operatorname{sstr}_{2}$ at low to medium levels (11); the human pancreatic islet cell carcinoma cells QGP1 (12), which express $\operatorname{sstr}_{2}$ at low levels; and the rat pancreatic acinar cells AR42J, which express $\operatorname{sstr}_{2}$ at high levels (13). We cultured all cells at $37^{\circ} \mathrm{C}$ and $5 \% \mathrm{CO}_{2}$ in Dulbecco modified Eagle medium or RPMI containing GlutaMAX (Thermo Fischer Scientific), $10 \%(\mathrm{v} / \mathrm{v})$ fetal bovine serum, penicillin $(100 \mathrm{U} / \mathrm{mL})$, and streptomy$\operatorname{cin}(100 \mu \mathrm{g} / \mathrm{mL})$.

\section{Radiolabeling}

We used the short half-life positron-emitter ${ }^{68} \mathrm{Ga}$ for biodistribution studies and PET imaging. We eluted ${ }^{68} \mathrm{Ga}$ from a commercially available ${ }^{68} \mathrm{Ge} /{ }^{68} \mathrm{Ga}$ generator and purified it as previously described (14). We then incubated the ${ }^{68} \mathrm{Ga}$ solution with DOTATOC at $0.1 \mathrm{mg} / \mathrm{mL}$ in Dulbecco phosphate-buffered saline at a ratio of $1: 1(\mathrm{v} / \mathrm{v})$ for $10 \mathrm{~min}$ at $95^{\circ} \mathrm{C}$. We determined the radiolabeling yield by radio-thin-layer chromatography and used only batches with radiochemical purities of greater than $97 \%$. 


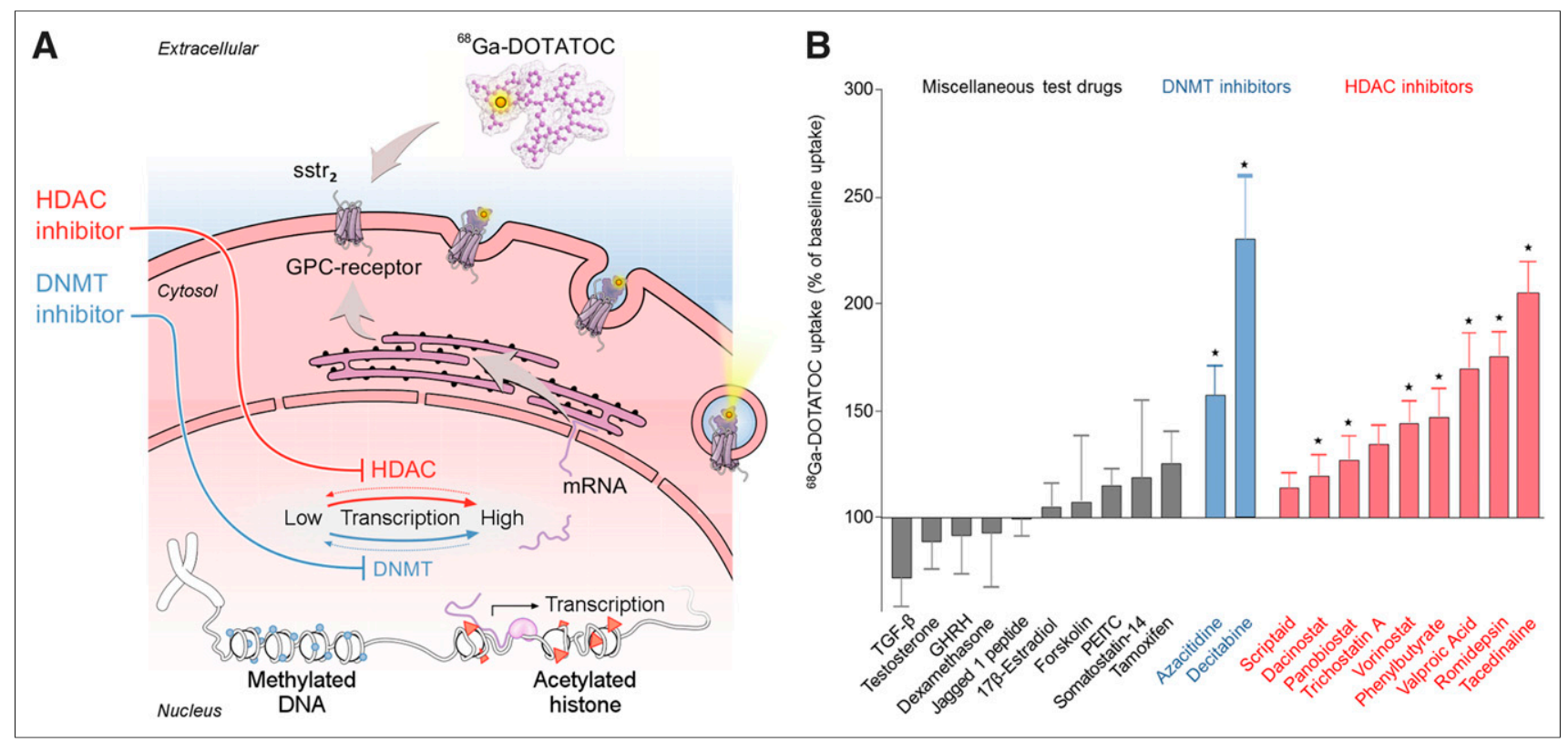

FIGURE 1. Screen revealed drugs that upregulate targets for molecular diagnosis and therapy. (A) Principle of drug screen: cells were tested for their baseline uptake of ${ }^{8} \mathrm{Ga}$-DOTATOC. Subsequently, they were treated with test drugs (Supplemental Table 1), including inhibitors of DNMT and HDAC. DNMT inhibitors and HDAC inhibitors reduce DNA methylation and stimulate histone acetylation, respectively, which induces upregulation of gene expression. Upregulation of target sstr $_{2}$ led to increased internalization of its ligand ${ }^{68} \mathrm{Ga}$-DOTATOC. Readout of screen was sstr ${ }_{2}$-mediated internalization of ${ }^{68} \mathrm{Ga}$-DOTATOC, monitored via its y-emission. (B) Drug-induced changes in ${ }^{68} \mathrm{Ga}$-DOTATOC uptake, normalized to baseline uptake (=100\%) in untreated cells. Statistical significance was tested using Student $t$ test and is indicated $\left({ }^{*} P<0.05\right)$. GHRH $=$ growth hormone-releasing hormone; PEITC $=$ phenethyl isothiocyanate; TGF- $\beta=$ transforming growth factor $\beta$.

\section{Quantitative Real-Time Polymerase Chain Reaction (qRT-PCR)}

We performed real-time qRT-PCR to determine messenger RNA levels using the primer Hs00265624_s1 for human $\mathrm{sstr}_{2}$, as previously described (15).

\section{Western Blotting}

We cultured cells with or without the test drug, and prepared cell lysates. Then, we analyzed the amount of $\operatorname{sstr}_{2}$ protein in the lysates via Western blot, using the sstr $_{2}$-specific antibody A01591 (Genscript \#A01591) in combination with the secondary antibody goat antirabbit IRDye 680 and goat antimouse IRDye 800 coupled to infrared dyes. We quantified all Western blots and coomassie-stained gels using a LICOR Odyssey scanner system.

\section{Enzyme-Linked Immunosorbent Assay (ELISA)}

We cultured cells with or without the test drugs and washed, fixed, and permeabilized them. Then, we assessed $\operatorname{sstr}_{2}$ expression using the primary $\operatorname{sstr}_{2}$-specific antibody UMB-1 and a secondary antibody goat antirabbit IgG horseradish peroxidase conjugate, by adding the horseradish peroxidase substrate and measuring the absorbance at $415 \mathrm{~nm}$. We normalized the specific absorbance to the total amount of protein and set the normalized specific absorbance value of the untreated cells to $100 \%$.

\section{Immunofluorescence Microscopy}

We cultured cells with or without the test drugs and washed, fixed, and permeabilized them. Then, we performed immunofluorescence microscopy as previously described (16), using the primary sstr ${ }_{2}$-specific antibody UMB-1 in combination with the secondary antibody Alexa Fluor 488 goat antirabbit IgG (H-L). Subsequently, we imaged the cells using a Nikon Eclipse TS 100 immunofluorescence microscope and a Nikon DS-Fil camera.

\section{Immunohistochemistry}

We processed BON-1 xenografts as conventional, 2- $\mu \mathrm{m}\left(\mathrm{sstr}_{2}\right)$ thick paraffin wax sections using the $\operatorname{sstr}_{2}$-specific primary antibody UMB-1. We performed immunohistochemical staining using the compact polymer dextran-peroxidase complex method (EnVision), which yields a brown staining signal (17). In addition, we used hemalum for counterstaining of the samples.

\section{Uptake Assay}

We performed all uptake experiments as previously described (18). In brief, we cultured cells with or without the test drugs and incubated them with ${ }^{68} \mathrm{Ga}$-DOTATOC. Subsequently we lysed the cells and determined the amount of intracellular ${ }^{68} \mathrm{Ga}$ using a $\gamma$-counter. We normalized the cell-associated radioactivity with the relative amount of protein reflecting the cell number and used untreated cells to measure the baseline uptake of radioactivity.

\section{Drug Screening}

To identify drugs that upregulate $\operatorname{sstr}_{2}$, we designed an in vitro screening based on cellular ${ }^{68} \mathrm{Ga}$-DOTATOC uptake as readout of $\mathrm{sstr}_{2}$ function (Fig. 1A). To increase the chance of clinically useful results, we included drugs that are already in clinical use and that have a potential to upregulate $\mathrm{sstr}_{2}$, for example, as they already demonstrated epigenetic modulation, especially of $s s t r_{2}$ gene expression. We screened all drugs at their therapeutic serum concentrations to facilitate potential clinical translation (Fig. 1B). We used BON-1 cells for screening and PC3 cells for validation.

\section{In Vitro Treatment}

We cultured cells with or without the test drugs and then incubated them with ${ }^{177} \mathrm{Lu}$-DOTATOC. After $2 \mathrm{~h}$, we washed and trypsinized the cells, reseeded them at 50,000 cells per milliliter, and cultivated them 


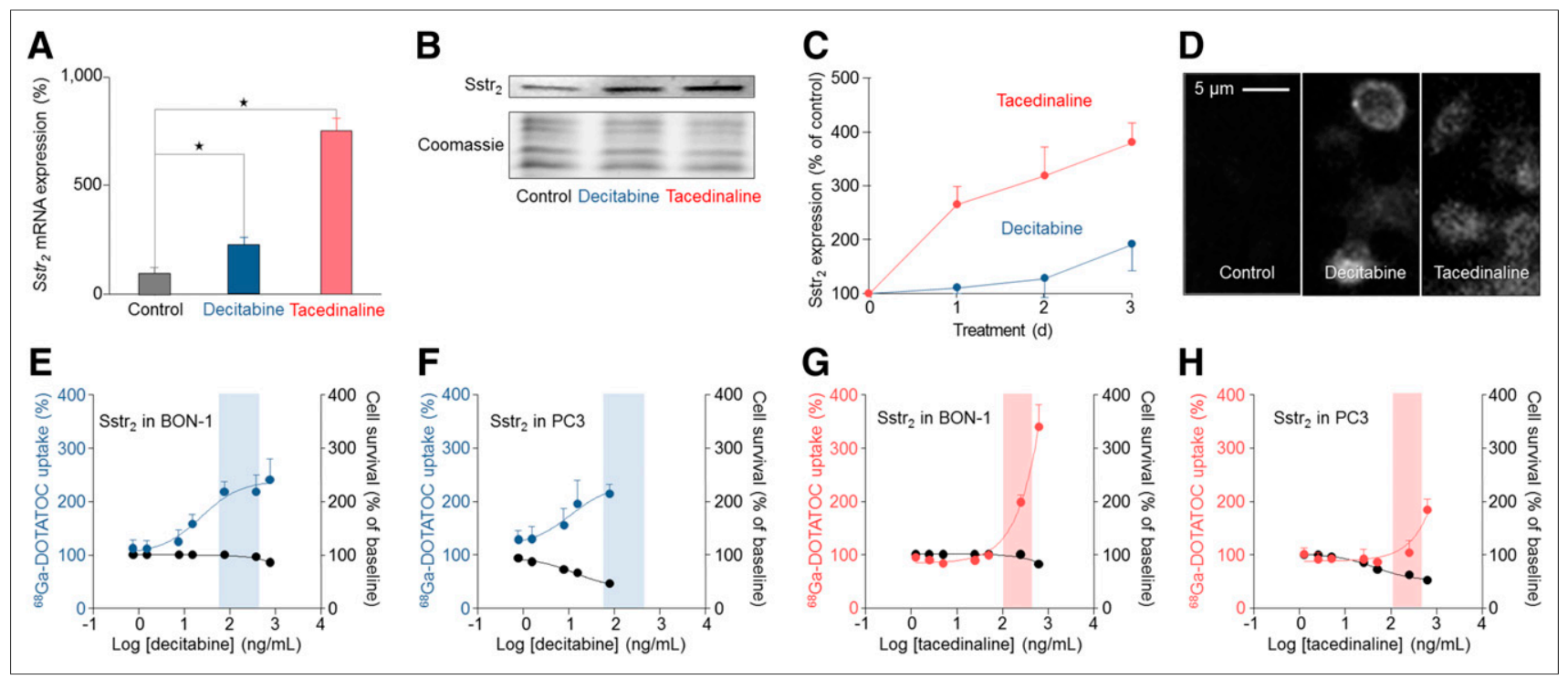

FIGURE 2. Drugs identified by screen improve molecular targeting. qRT-PCR and Western blot, ELISA, and immunocytochemistry with sstr ${ }_{2}{ }^{-}$ specific antibodies were performed in BON-1 and PC3 cells to assess effects of decitabine and tacedinaline on sstr ${ }_{2}$ expression versus untreated controls. Decitabine and tacedinaline were tested at their therapeutic serum concentrations. Cell survival and uptake assays at different drug concentrations were performed to assess dose-dependent toxicity and changes in sstr $_{2}$ expression. All results are expressed as changes in percentage and normalized to results in untreated cells. (A) qRT-PCR showing sstr ${ }_{2}$ messenger RNA expression in untreated BON-1 cells and BON-1 cells treated with decitabine $(75 \mathrm{ng} / \mathrm{mL})$ or tacedinaline $(500 \mathrm{ng} / \mathrm{mL})$. (B) Western blot showing sstr ${ }_{2}$ expression in untreated BON-1 cells and BON-1 cells treated with decitabine $(75 \mathrm{ng} / \mathrm{mL})$ or tacedinaline $(500 \mathrm{ng} / \mathrm{mL})$. Coomassie staining displays remaining protein after blotting. (C) ELISA showing sstr $_{2}$ expression over a time period of 1,2 , and $3 \mathrm{~d}$ in BON-1 cells treated with decitabine $(75 \mathrm{ng} / \mathrm{mL})$ or tacedinaline $(500 \mathrm{ng} / \mathrm{mL})$. (D) Immunocytochemistry showing sstr ${ }_{2}$ expression in untreated BON-1 cells and BON-1 cells treated with decitabine $(75 \mathrm{ng} / \mathrm{mL})$ or tacedinaline $(500 \mathrm{ng} / \mathrm{mL})$ for $3 \mathrm{~d}$. Dose-dependent effects of decitabine on uptake of $\mathrm{sstr}_{2}$ ligand ${ }^{68} \mathrm{Ga}-\mathrm{DOTATOC}$ in BON-1 cells (E) and PC3 cells (F). Dosedependent effects of tacedinaline on uptake of $s \operatorname{st}_{2}$ ligand ${ }^{68} \mathrm{Ga}-D O T A T O C$ in BON-1 cells $(\mathrm{G})$ and PC3 cells $(\mathrm{H})$. Therapeutic serum concentration of decitabine $(65-460 \mathrm{ng} / \mathrm{mL})$ and tacedinaline $(135-570 \mathrm{ng} / \mathrm{mL})$ is indicated by highlighted areas. Dose-dependent cell survival representing toxicity of decitabine and tacedinaline is shown as black curves.

for an additional $12 \mathrm{~h}$. Then, we lysed the cells and determined the amount of protein reflecting the number of cells.

\section{Animal Studies}

We treated Nude-Foxn $1^{\text {nu }}$ mice carrying BON-1 or PC3 xenografts with decitabine or phosphate-buffered saline over a period of $9 \mathrm{~d}$. Then, we injected $10 \mathrm{MBq}$ of ${ }^{68} \mathrm{Ga}$-DOTATOC via the tail vain and performed PET/CT imaging or biodistribution studies $1 \mathrm{~h}$ later. For the latter, we collected all organs, measured the uptake of ${ }^{68} \mathrm{Ga}$-DOTATOC via a $\gamma$-counter, and computed all organ activity concentrations as percentage decay-corrected injected activity per gram of tissue. All animal experiments were conducted after approval by the local authorities and in accordance with the national regulations for animal treatment.

\section{Statistical Analysis}

We expressed all data as mean $\pm \mathrm{SD}$ and compared results for 2 or more groups via the Student $t$ test or ANOVA, respectively, using SPSS 21. We established all dose-effect curves using the Boltzmann method in Prism 5. We considered $P$ values of less than 0.05 to indicate statistical significance.

\section{RESULTS}

The screen identified the inhibitor of DNA methyltransferase (DNMT) decitabine and the inhibitors of histone deacetylase (HDAC) tacedinaline and romidepsin as most efficacious for upregulating $\mathrm{sstr}_{2}$ in BON-1 cells (Fig. 1B; Supplemental Fig. 1).

\section{Drugs Identified by Screen Improve Molecular Targeting}

We tested the effects of these drugs on the transcription, translation, and function of $\operatorname{sstr}_{2}$ (Fig. 2). In line with the screening results, qRT-PCR analysis demonstrated that decitabine and tacedinaline increased $s s r_{2}$ gene expression (Fig. 2A), Western blot confirmed upregulation of $\operatorname{sstr}_{2}$ protein expression (Fig. 2B), ELISA showed an increase in $\operatorname{sstr}_{2}$ expression over time (Fig. 2C), and immunocytochemistry confirmed membrane localization of upregulated $\operatorname{sstr}_{2}$ (Fig. 2D). We then evaluated the effects of decitabine on $\operatorname{sstr}_{2}$ function and cell toxicity in various cell models. Decitabine increased ${ }^{68} \mathrm{Ga}$-DOTATOC uptake within its therapeutic serum concentration in BON-1 and PC3 (Figs. 2E and 2F), whereas it had no significant effect on $\operatorname{sstr}_{2}$ function in QGP1 and AR42J cells (Supplemental Fig. 2).

We then assessed whether tacedinaline has effects similar to those of decitabine on $\operatorname{sstr}_{2}$ function and cell toxicity. In BON-1, tacedinaline induced a dose-dependent increase of ${ }^{68} \mathrm{Ga}$-DOTATOC uptake, albeit to lower levels than those achieved with decitabine, and without reaching a plateau within the therapeutic serum concentration (Fig. 2G).

In PC3, tacedinaline at therapeutic serum concentrations had no significant effect on ${ }^{68} \mathrm{Ga}$-DOTATOC uptake (Fig. $2 \mathrm{H}$ ). Overall, tacedinaline showed toxicity similar to decitabine in BON-1 (Figs. 2E and 2G) and lower toxicity in PC3 (Figs. 2F and 2H). Decitabine and tacedinaline upregulated $\mathrm{sstr}_{2}$ with similar efficacy, whereas tacedinaline treatment in PC3 did not upregulate $\operatorname{sstr}_{2}$. 


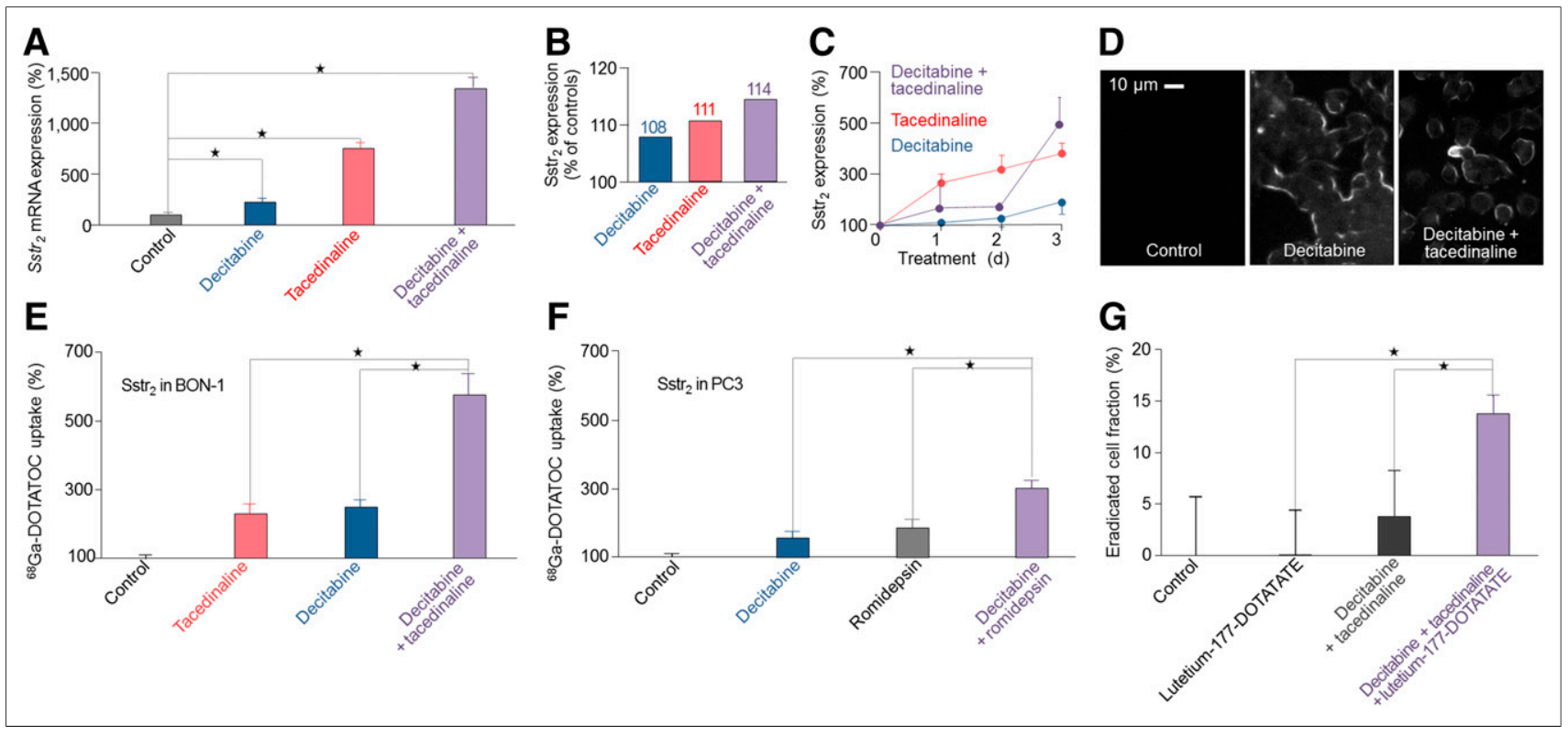

FIGURE 3. Combination treatment is superior to single-drug treatment. qRT-PCR and Western blot, ELISA, and immunocytochemistry with sstr ${ }_{2}-$ specific antibodies were performed to assess effects of combination treatment on sstr ${ }_{2}$ messenger RNA and sstr ${ }_{2}$ expression compared with effects of single-drug treatment. Uptake assays were performed to assess changes in sstr $_{2}$ expression for single-drug treatment and combination treatment. All results are expressed as changes in percentage and normalized to results in untreated cells. (A) qRT-PCR showing sstr ${ }_{2}$ messenger RNA expression in untreated BON-1 cells and BON-1 cells treated with decitabine $(75 \mathrm{ng} / \mathrm{mL})$, tacedinaline $(500 \mathrm{ng} / \mathrm{mL})$, or decitabine $(75 \mathrm{ng} / \mathrm{mL}) \mathrm{plus}$ tacedinaline $(500 \mathrm{ng} / \mathrm{mL})$. (B) Quantification of Western blot showing sstr ${ }_{2}$ expression in untreated BON-1 cells and BON-1 cells treated with decitabine $(75 \mathrm{ng} / \mathrm{mL})$, tacedinaline $(500 \mathrm{ng} / \mathrm{mL})$, or decitabine $(75 \mathrm{ng} / \mathrm{mL})$ plus tacedinaline $(500 \mathrm{ng} / \mathrm{mL})$ for $3 \mathrm{~d}$. (C) ELISA showing sstr ${ }_{2}$ expression over time period of 1,2 , and $3 \mathrm{~d}$ in BON-1 treated with decitabine $(75 \mathrm{ng} / \mathrm{mL})$, tacedinaline $(500 \mathrm{ng} / \mathrm{mL})$, or decitabine $(75 \mathrm{ng} / \mathrm{mL})$ plus tacedinaline $(500 \mathrm{ng} / \mathrm{mL})$. (D) Immunocytochemistry showing sstr ${ }_{2}$ in untreated BON-1 cells and BON-1 cells treated with decitabine $(75 \mathrm{ng} / \mathrm{mL})$ alone and with

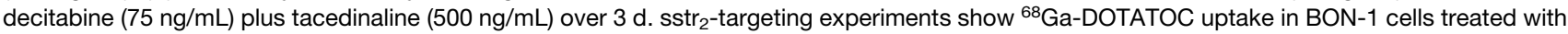
decitabine, tacedinaline, and their combination (E) and in PC3 cells treated with decitabine, romidepsin, and their combination (F). (G) Treatment studies assessing eradication fraction in untreated BON-1 cells, BON-1 cells treated with $5 \mathrm{MBq} / \mathrm{mL}$ of ${ }^{177}$ Lu-DOTATATE, BON-1 cells treated with decitabine plus tacedinaline, and BON-1 cells treated with ${ }^{177} \mathrm{Lu}$-DOTATATE after sstr $_{2}$ upregulation with decitabine plus tacedinaline. Statistical significance was tested using Student $t$ test and is indicated ( $\left.{ }^{\star} P<0.05\right)$.

\section{Combination Treatment Is Superior to Single-Drug Treatment}

Because the drugs identified by the screen have different mechanisms of action, we evaluated potential synergistic effects to increase the efficacy of target upregulation (Fig. 3). qRTPCR analysis revealed upregulation of $s s t_{2}$ gene expression by decitabine plus tacedinaline (Fig. 3A), with stronger effects than those of either drug alone (Fig. 2A). Western blot confirmed upregulation of $\mathrm{sstr}_{2}$ protein (Fig. 3B); ELISA showed increased $\operatorname{sstr}_{2}$ expression over time (Fig. 3C), with stronger effects than those of either drug alone (Fig. 2C); and immunocytochemistry confirmed membrane localization of upregulated $\operatorname{sstr}_{2}$ (Fig. 3D).

Subsequently, we tested for the most efficacious combinations of drugs identified by the screen on the functional level and found that decitabine, tacedinaline, and romidepsin upregulation of $\operatorname{sstr}_{2}$ depended on the cell model. In BON-1, decitabine plus tacedinaline was most efficacious in upregulating $\mathrm{sstr}_{2}$, superior to either single drug (Fig. 3E). Conversely, in PC3, tacedinaline as a single drug or in combination treatments had no effects on sstr $_{2}$ function. In these cells, the most efficacious combination was decitabine plus romidepsin (Fig. 3F). Next, we studied whether sstr $_{2}$ upregulation with decitabine plus tacedinaline can improve sstr $_{2}$-targeted therapy with ${ }^{177} \mathrm{Lu}$-DOTATATE. In BON-1 cells, ${ }^{177} \mathrm{Lu}$-DOTATATE had no detectable effects without pretreatment; however, we found significant effects after pretreatment with decitabine plus tacedinaline (Fig. 3G).

\section{Target Upregulation Improves Molecular Imaging In Vivo}

We then evaluated the observed effects in vivo. We used decitabine, which was the most efficacious drug in the in vitro experiments (Fig. 4). Immunohistochemistry of BON-1 tumors in athymic nude mice undergoing systemic decitabine treatment confirmed upregulation of $\operatorname{sstr}_{2}$ protein (Fig. 4A). On the functional level, decitabine significantly and dose-dependently increased ${ }^{68} \mathrm{Ga}$-DOTATOC uptake in both human cancer models, BON-1 and PC3 (Fig. 4B).

We confirmed sstr $_{2}$-mediated effects in vivo by performing blocking experiments, which significantly reduced ${ }^{68} \mathrm{Ga}$-DOTATOC uptake in tumors and organs with high $\operatorname{sstr}_{2}$ expression, for example, the pancreas and stomach (Fig. 4B) (19). In contrast, the blocking did not decrease the kidney uptake. Importantly, in both tumor models, decitabine improved the overall biodistribution (Supplemental Fig. 5B), especially by increasing the tumor-to-background (Fig. 4C) and tumor-to-kidney ratio (Fig. 4D), thereby indicating its potential for improving tumor detectability and treatability.

Finally, we used receptor-targeted imaging with ${ }^{68} \mathrm{Ga}$-DOTATOC to visualize decitabine-based $\mathrm{sstr}_{2}$ upregulation in vivo. Decitabine increased tumor uptake and tumor-to-background ratio and thereby converted almost undetectable BON-1 tumors into detectable tumors (PET, Fig. 4E; PET/CT, Supplemental Fig. 5A).

\section{DISCUSSION}

Our screen identified the DNMT inhibitor decitabine and the HDAC inhibitors romidepsin and tacedinaline as relevant modulators 


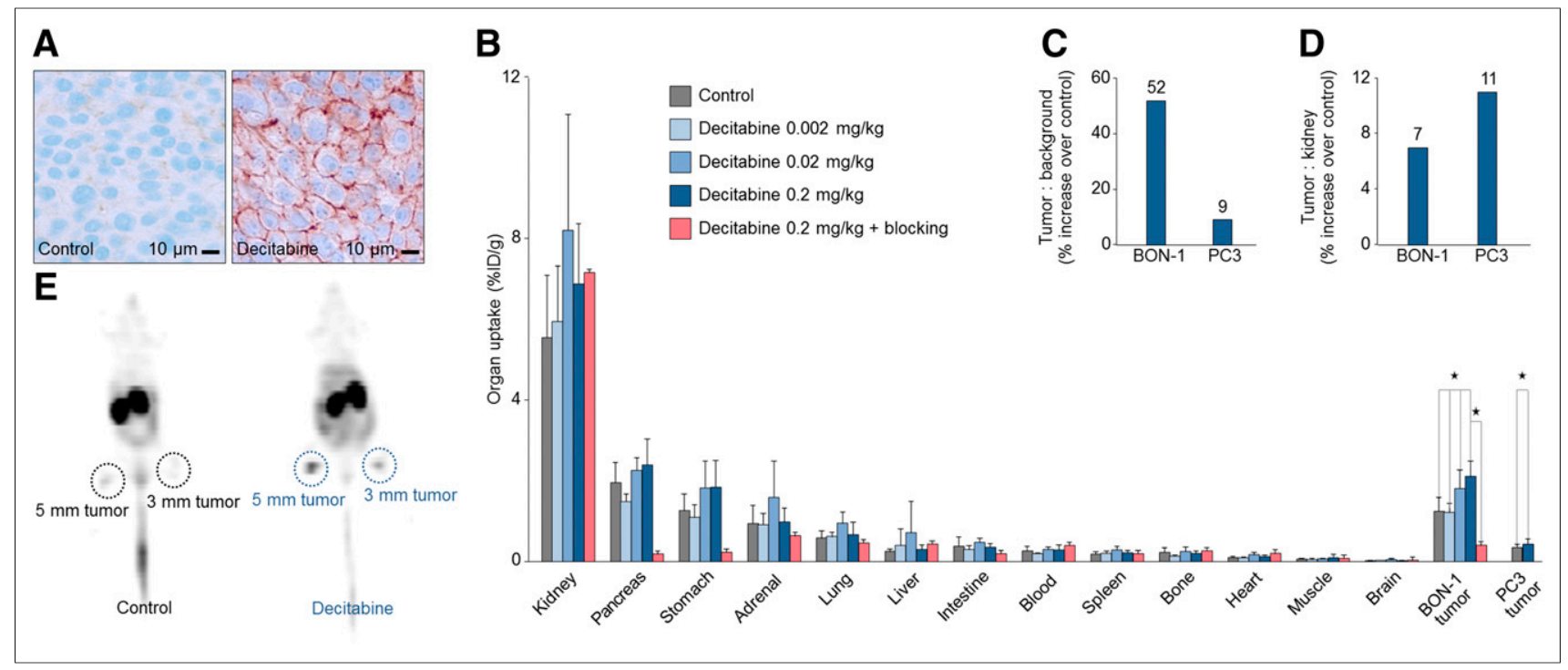

FIGURE 4. Target upregulation improves molecular imaging in vivo. In vivo experiments were performed in BON-1 and PC3 tumor-bearing nude mice. Mice received subcutaneous injections of decitabine over 9 d. (A) Immunohistochemistry with UMB-1 antisstr ${ }_{2}$ monoclonal antibody in BON-1 tumors with and without decitabine treatment $(n=4)$. (B) Biodistribution studies of ${ }^{68} \mathrm{Ga}-\mathrm{DOTATOC}$ uptake in all relevant organs, BON-1 tumors $(n=21)$, and PC3 tumors $(n=30)$. Dose-escalation studies were performed with $0.002,0.02$, and $0.2 \mathrm{mg} / \mathrm{kg}$ decitabine $(n=20)$. To evaluate specific sstr ${ }_{2}$ binding blocking experiments were performed by coapplication of excess of nonradiolabeled DOTATOC to compete with ${ }^{68} \mathrm{Ga}$-DOTATOC binding $(n=5)$. (C) Tumor-to-background ratios of ${ }^{8} \mathrm{Ga-DOTATOC}$ uptake with and without decitabine treatment in BON-1 tumors, representing key factor for imaging contrast in sstr $_{2}$-targeted imaging $(n=21)$. (D) Tumor-to-kidney ratios of ${ }^{68} \mathrm{Ga}$-DOTATOC uptake with and without decitabine treatment in BON-1 tumors, representing measure of therapeutic index in sstr ${ }_{2}$-targeted radiopeptide therapy $(n=30)$. (E) ${ }^{68} \mathrm{Ga}-\mathrm{DOTATOC}$ PET with and without decitabine treatment. Statistical significance was tested using Student $t$ test and ANOVA and is indicated $\left({ }^{*} P<0.05\right)$.

of $\operatorname{sstr}_{2}$ expression. Tacedinaline recently underwent clinical phase 3 testing, whereas decitabine and romidepsin are already in clinical routine for treating myelodysplastic syndrome and T-cell lymphoma (20-22). All 3 drugs are known to modulate cellular transcriptional activity. Specifically, DNMT inhibitors enhance gene transcription by inducing DNA hypomethylation, whereas HDAC inhibitors enhance transcriptional activity by increasing histone acetylation $(23,24)$.

Epigenetic modulation has a high clinical potential (25), yet little is known about the epigenetic control of $\operatorname{sstr}_{2}$. Previous studies suggested effects on a transcriptional level via DNMT and HDAC inhibition $(26,27)$ and on a translational level via HDAC inhibition (28). In the present study, we confirmed these findings and expanded the principle to the functional level in vitro and in vivo. Thereby, we used BON-1 and PC3 cells, which represent a clinical scenario of low $\mathrm{sstr}_{2}$ expression and low ${ }^{68} \mathrm{Ga}$-DOTATOC uptake. In addition, we used AR42J cells, which had high sstr $_{2}$ expression, and QGP1 cells, which had an undetectable sstr $_{2}$ expression. BON-1, AR42J, and QGP1 are commonly used neuroendocrine tumor models, whereas PC3 is a commonly used prostate cancer model with expression of $\operatorname{sstr}_{2}(11)$.

The present study indicates that the potential for improving targeted approaches with DNMT and HDAC inhibitors might vary considerably among different tumors. DNMT inhibition upregulated $\mathrm{sstr}_{2}$ in BON-1 and in PC3, whereas HDAC inhibition upregulated $\operatorname{sstr}_{2}$ in BON-1 but not in PC3. However, DNMT and HDAC inhibition had no significant effects in AR42J and QGP1. Consequently, epigenetic upregulation of $\operatorname{sstr}_{2}$ might have the highest potential in tumors with a measurable but not already maximally amplified target expression.
Furthermore, epigenetic upregulation of drug targets might benefit from a combination of DNMT and HDAC inhibition. The combined use of DNMT and HDAC inhibitors already demonstrated antitumor effects superior to those of single drug treatment (20), and similarly, we found synergistic effects for their application as epigenetic modifiers. These synergistic effects largely depended on the tumor model and target molecule, which is in line with the clinical observation that cancer treatment with DNMT and HDAC inhibitors shows varying efficiency among tumor types (29). The present findings indicate that target upregulation might require tailoring to tumor type, tumor characteristics, and target molecule to optimize the efficacy of our approach.

The feasibility of our approach could be most efficiently tested with a low-dose regimen of a single drug, for example, decitabine, in patients with progressive metastasized neuroendocrine tumors that demonstrate low $\operatorname{sstr}_{2}$ expression and low ${ }^{68} \mathrm{Ga}$-DOTATATE uptake. Such a translational study should use ${ }^{68} \mathrm{Ga}$-DOTATATE PET before and after decitabine treatment to verify functional upregulation of $\operatorname{sstr}_{2}$ and thereby stratify patients to ${ }^{177} \mathrm{Lu}$-DOTATATE treatment after DNMT inhibition.

A clinical study would be feasible and safe because of the established use of decitabine and ${ }^{68} \mathrm{Ga}$-DOTATATE. Furthermore, clinical translation would be promising due to the improvements of tumor-to-background and tumor-to-kidney ratios, which are key elements for successful targeted imaging and treatment and which significantly exceed the improvements that the most frequently used $\mathrm{sstr}_{2}$ radioligand DOTATATE provided over its predecessors (18). Successful clinical translation of epigenetic target upregulation might provide patients with neuroendocrine tumors with low 
$\operatorname{sstr}_{2}$ expression, who have failed all currently available treatments, with new therapeutic options.

Finally, successful translation has a potential impact beyond sstr $_{2}$ targeting, because reversible DNA hypermethylation has been described for promoters of clinically relevant target structures other than $\operatorname{sstr}_{2}(27)$, including the sodium-iodine symporter in differentiated thyroid cancer (30), CD-20 in lymphoma (31), and human epidermal growth factor receptor 2 in breast cancer (32). Thus, drug-based epigenetic modulation is a promising strategy to facilitate molecularly targeted diagnostics and therapies for a wide range of oncologic patients.

\section{CONCLUSION}

We present an approach that uses epigenetic modifiers to improve sstr $_{2}$ targeting in vitro and in vivo. The presented method is easily transferrable into the clinic and might provide patients who have failed various treatment regimens with new therapeutic options.

\section{DISCLOSURE}

The costs of publication of this article were defrayed in part by the payment of page charges. Therefore, and solely to indicate this fact, this article is hereby marked "advertisement" in accordance with 18 USC section 1734. Our work was supported by the OPO-Foundation, Braun-Foundation, Gebauer-Foundation, and Stiftung zur Krebsbekämpfung. Moreover, Martin A. Walter (31003A_156731) and Aurel Perren (310030_144236) were supported by the Swiss National Science Foundation. No other potential conflict of interest relevant to this article was reported.

\section{ACKNOWLEDGMENTS}

We thank Ramtin Khalafi for technical assistance, Andrew Q. Tran for medical illustrating assistance, and Duncan Marriott and Caroline Spencer (Rx Communications, Mold, U.K.) for medical writing assistance, which was funded by the University Hospital Bern. Finally, we thank Nadia Sanchez, Rebecca Dumont, and Johannes Czernin for the valuable discussions and helpful comments on the manuscript.

\section{REFERENCES}

1. Caplin ME, Pavel M, Ruszniewski P. Lanreotide in metastatic enteropancreatic neuroendocrine tumors. $N$ Engl J Med. 2014;371:1556-1557.

2. Otte A, Herrmann R, Heppeler A, et al. Yttrium-90 DOTATOC: first clinical results. Eur J Nucl Med. 1999;26:1439-1447.

3. Marincek N, Radojewski P, Dumont RA, et al. Somatostatin receptor-targeted radiopeptide therapy with ${ }^{90}$ Y-DOTATOC and ${ }^{177} \mathrm{Lu}$-DOTATOC in progressive meningioma: long-term results of a phase II clinical trial. J Nucl Med. 2015;56: 171-176.

4. Oberg K. Molecular imaging radiotherapy: theranostics for personalized patient management of neuroendocrine tumors (NETs). Theranostics. 2012;2:448-458.

5. Schraml C, Schwenzer NF, Sperling O, et al. Staging of neuroendocrine tumours: comparison of $\left[{ }^{68} \mathrm{Ga}\right] \mathrm{DOTATOC}$ multiphase PET/CT and whole-body MRI. Cancer Imaging. 2013;13:63-72.

6. Sadowski SM, Neychev V, Millo C, et al. Prospective study of ${ }^{68} \mathrm{Ga}$-DOTATATE positron emission tomography/computed tomography for detecting gastroentero-pancreatic neuroendocrine tumors and unknown primary sites. J Clin Oncol. 2016;34:588-596.

7. Villard L, Romer A, Marincek N, et al. Cohort study of somatostatin-based radiopeptide therapy with $\left[{ }^{90} \mathrm{Y}-\mathrm{DOTA}\right]-\mathrm{TOC}$ versus $\left[{ }^{90} \mathrm{Y}\right.$-DOTA]-TOC plus [ $\left.{ }^{177} \mathrm{Lu}-\mathrm{DOTA}\right]-\mathrm{TOC}$ in neuroendocrine cancers. J Clin Oncol. 2012;30:11001106.
8. Appetecchia M, Baldelli R. Somatostatin analogues in the treatment of gastroenteropancreatic neuroendocrine tumours, current aspects and new perspectives. J Exp Clin Cancer Res. 2010;29:19.

9. Buscail L, Saint-Laurent N, Chastre E, et al. Loss of sst2 somatostatin receptor gene expression in human pancreatic and colorectal cancer. Cancer Res. 1996;56: 1823-1827.

10. Cariaga-Martinez AE, Lorenzati MA, Riera MA, et al. Tumoral prostate shows different expression pattern of somatostatin receptor 2 (SSTR2) and phosphotyrosine phosphatase SHP-1 (PTPN6) according to tumor progression. Adv Urol. 2009:723831.

11. Sun LC, Mackey LV, Luo J, Fuselier JA, Coy DH. Targeted chemotherapy using a cytotoxic somatostatin conjugate to inhibit tumor growth and metastasis in nude mice. Clin Med Oncol. 2008;2:491-499.

12. Doihara H, Nozawa K, Kojima R, Kawabata-Shoda E, Yokoyama T, Ito H. QGP1 cells release 5-HT via TRPA1 activation: a model of human enterochromaffin cells. Mol Cell Biochem. 2009;331:239-245.

13. Lima MJ, Docherty HM, Chen Y, Docherty K. Efficient differentiation of AR42J cells towards insulin-producing cells using pancreatic transcription factors in combination with growth factors. Mol Cell Endocrinol. 2012;358:69-80.

14. Zhernosekov KP, Filosofov DV, Baum RP, et al. Processing of generator-produced ${ }^{68} \mathrm{Ga}$ for medical application. J Nucl Med. 2007;48:1741-1748.

15. Ben-Shlomo A, Pichurin O, Barshop NJ, et al. Selective regulation of somatostatin receptor subtype signaling: evidence for constitutive receptor activation. Mol Endocrinol. 2007;21:2565-2578.

16. Cescato R, Schulz S, Waser B, et al. Internalization of sst2, sst3, and sst5 receptors: effects of somatostatin agonists and antagonists. J Nucl Med. 2006;47: 502-511.

17. Sabattini E, Bisgaard K, Ascani S, et al. The EnVision ++ system: a new immunohistochemical method for diagnostics and research-critical comparison with the APAAP, ChemMate, CSA, LABC, and SABC techniques. J Clin Pathol. 1998;51:506-511.

18. Storch D, Behe M, Walter MA, et al. Evaluation of [ $\left.{ }^{99 \mathrm{~m}} \mathrm{Tc} / \mathrm{EDDA} / \mathrm{HYNIC0}\right]$ octreotide derivatives compared with $\left[{ }^{111}\right.$ In-DOTA0,Tyr3, Thr8] octreotide and [ ${ }^{111}$ In-DTPA0]octreotide: does tumor or pancreas uptake correlate with the rate of internalization? J Nucl Med. 2005;46:1561-1569.

19. Yamada Y, Post SR, Wang K, Tager HS, Bell GI, Seino S. Cloning and functional characterization of a family of human and mouse somatostatin receptors expressed in brain, gastrointestinal tract, and kidney. Proc Natl Acad Sci USA. 1992;89:251-255.

20. Garcia-Manero G. Myelodysplastic syndromes: 2014 update on diagnosis, riskstratification, and management. Am J Hematol. 2014;89:97-108.

21. Barbarotta L, Hurley K. Romidepsin for the treatment of peripheral T-cell lymphoma. J Adv Pract Oncol. 2015;6:22-36.

22. Perabo FG, Muller SC. New agents for treatment of advanced transitional cell carcinoma. Ann Oncol. 2007;18:835-843.

23. Martínez-Iglesias O, Ruiz-Llorente L, Sanchez-Martinez R, Garcia L, Zambrano A, Aranda A. Histone deacetylase inhibitors: mechanism of action and therapeutic use in cancer. Clin Transl Oncol. 2008;10:395-398.

24. Momparler RL. Epigenetic therapy of cancer with 5-aza-2'-deoxycytidine (decitabine). Semin Oncol. 2005;32:443-451.

25. Dear AE. Epigenetic modulators and the new immunotherapies. $N$ Engl J Med. 2016;374:684-686.

26. Liu Z, Marquez M, Nilsson S, Holmberg AR. Incubation with somatostatin, 5-aza decitabine and trichostatin up-regulates somatostatin receptor expression in prostate cancer cells. Oncol Rep. 2008;20:151-154.

27. Torrisani J, Hanoun N, Laurell $\mathrm{H}$, et al. Identification of an upstream promoter of the human somatostatin receptor, hSSTR2, which is controlled by epigenetic modifications. Endocrinology. 2008;149:3137-3147.

28. Sun L, Qian Q, Sun G, et al. Valproic acid induces NET cell growth arrest and enhances tumor suppression of the receptor-targeted peptide-drug conjugate via activating somatostatin receptor type II. J Drug Target. 2016;24:169-177.

29. Mack GS. To selectivity and beyond. Nat Biotechnol. 2010;28:1259-1266.

30. Venkataraman GM, Yatin M, Marcinek R, Ain KB. Restoration of iodide uptake in dedifferentiated thyroid carcinoma: relationship to human $\mathrm{Na}+/ \mathrm{I}$-symporter gene methylation status. J Clin Endocrinol Metab. 1999;84:2449-2457.

31. Ushmorov A, Leithauser F, Sakk O, et al. Epigenetic processes play a major role in B-cell-specific gene silencing in classical Hodgkin lymphoma. Blood. 2006;107: 2493-2500.

32. Huang Y, Fu P, Fan W. Novel targeted therapies to overcome trastuzumab resistance in HER2-overexpressing metastatic breast cancer. Curr Drug Targets. 2013;14:889-898. 